\title{
Wie Banken die Produktivität steigern
}

\author{
Hannah Winterberg
}

\section{Relevanz}

Damit sich Innovation entfalten kann, muss Strukturwandel stattfinden. Innovative Unternehmen wachsen stark, andere schrumpfen oder scheiden aus. Investitionskapital soll von wenig profitablen Verwendungen abgezogen werden und dorthin fließen, wo die Wertschöpfung hoch und die Perspektiven gut sind. Das steigert die Produktivität und stärkt das Wachstum. Mit der Kreditwürdigkeitsprüfung und laufenden Überwachung lenken die Banken die Kreditvergabe auf profitable Bereiche, wo die Rückzahlung ziemlich sicher ist. Kreditlinien nicht zu verlängern oder ganz abzuschreiben, wenn die Kreditwürdigkeit nicht mehr gegeben ist, hält Finanzierung von unprofitablen Verwendungen fern. Dazu brauchen Banken eine robuste Kapitalausstattung und müssen frei von staatlichen Interventionen sein, die oft auf den Erhalt von existierenden anstatt auf die Schaffung neuer Arbeitsplätze ausgerichtet sind und strukturkonservierend wirken.

\section{Christian Keuschnigg}

\section{Quelle}

Der nachfolgende Text ist eine Zusammenfassung von: Betrand, Marianne, Antoinette Schoar und David Thesmar (2007), Banking Deregulation and Industry Structure: Evidence from the French Banking Reforms of 1985, The Journal of Finance 62(2), 597-628.

\section{H. Winterberg $(\bowtie)$}

Universität St. Gallen, St. Gallen, Schweiz

E-Mail: hannah.winterberg@student.unisg.ch 
Ein leistungsfähiger Finanzsektor und eine schnell wachsende Wirtschaft gehen häufig Hand in Hand. Es ist jedoch schwierig, die Richtung der Kausalität zwischen Wirtschaftswachstum und der Entwicklung des Finanzsektors empirisch einwandfrei zu ermitteln. Die Autoren nutzen hierzu ein sogenanntes „,natürliches Experiment", nämlich die Deregulierung des französischen Bankensektors in den 1980er Jahren, welche die Kreditvergabe von Banken entscheidend verändert hat. Um den Effekt der Deregulierung isoliert betrachten zu können, vergleichen sie die Entwicklung von Unternehmen in besonders bankabhängigen Branchen mit anderen Unternehmen, welche sich weniger stark über Bankenkredite finanzieren. Bei ihrer Analyse nutzen die Autoren einen sehr umfangreichen Datensatz der französischen Steuerbehörden mit Informationen zu rund 15.000 börsennotierten und privaten Unternehmen.

Der französische Bankensektor war bis Mitte der 1980er Jahre stark von staatlichen Eingriffen geprägt. Es existierte ein Netz staatlich bevorzugter Banken, welche subventionierte Kredite an Unternehmen vergaben, die als besonders förderungswürdig galten. Dabei wurde die staatlich organisierte Vergabe von subventionierten Krediten häufig zum Erhalt von Arbeitsplätzen genutzt. Schließlich wurden mehrere Institute verstaatlicht. Im Jahr 1979 war gut die Hälfte aller Kredite subventioniert und die Banken wiesen einen immer größer werdenden Anteil an notleidenden Krediten in ihren Bilanzen aus.

$\mathrm{Ab} 1985$ wurde der französische Finanzsektor umfassend reformiert. Die staatliche Steuerung und Subventionierung von Krediten wurde abgeschafft, einige Banken privatisiert und die Geldpolitik weg von einer Kontrolle der Kreditvergabe hin zu einer Zinspolitik neu ausgerichtet. Fortan sollten die Kapitalflüsse durch den Markt gesteuert werden, wobei es bestimmte Reserveverpflichtungen zu beachten gab. Die Deregulierung der französischen Banken in den späten 1980er Jahren hat den Bankensektor von einem durch staatliche Intervention geprägten hin zu einem marktwirtschaftlichen System verwandelt.

Die Deregulierung des Bankensektors führte zu positiven Veränderungen im Finanzsystem wie auch in der Wirtschaft. Die Reform hatte Auswirkungen auf die Kreditvergabe der Banken und dadurch auf die Kapitalstruktur der Unternehmen. Zudem schuf sie einen Anreiz für Unternehmen, produktiver zu werden, und verstärkte die Dynamik von Unternehmensgründungen und Schließungen. Letztlich verringerte die Reform tendenziell auch die Marktkonzentration von Unternehmen bzw. deren Marktmacht.

Nach der Reform sind Banken weniger bereit, schlecht gehende Unternehmen zu retten. Stattdessen unterstützen sie profitable Unternehmen während einer Krise durch zusätzliche Kredite. 
Im Zuge der Deregulierung erlangten die Banken wieder Autonomie über ihre Kreditvergabe, wodurch sich die Effizienz verbesserte. Dies zeigt sich insbesondere darin, dass die Banken ihre Entscheidungen stärker an der Ertragsfähigkeit der Kreditnehmer ausrichteten. Vor der Reform erhielten auch unprofitable Unternehmen weitere Kredite, wenn sie in die Krise geraten waren. Nach der Reform gingen die Banken in solchen Fällen wesentlich restriktiver vor und waren oft nicht mehr bereit, schwache Unternehmen zu retten. Gleichzeitig führte die Reform zu einer stärkeren Kreditvergabe an grundsätzlich profitable Unternehmen, die auch während einer Krise weiter unterstützt wurden. Die Fähigkeit der Banken, ein erfolgreiches Geschäftsmodell zu erkennen, verbesserte sich. Vor der Reform wiesen Unternehmen nach einer Kreditaufnahme im Schnitt eine niedrigere Rentabilität auf als vorher. Dieser Zusammenhang wurde durch die Reform umgedreht. Seither ist die Kreditvergabe an ein Unternehmen ein Indikator für einen zu erwartenden Anstieg der Rentabilität.

Betrug der Verschuldungsgrad der Unternehmen vor der Reform des Bankensektors noch im Schnitt $79 \%$, lag er danach bei $69 \%$. Dabei entfiel ein Großteil des Rückgangs auf den Abbau von Bankkrediten (-6 Prozentpunkte). Die Handelskredite sanken um 4 Prozentpunkte, während das Eigenkapital um 8 Prozentpunkte stieg.

Die geänderte Kreditvergabepolitik der Banken spiegelte sich auch in der Kapitalstruktur der Unternehmen wieder. Branchen, welche vor der Reform besonders stark von der Kreditfinanzierung der Banken abhängig waren und tendenziell stärker von subventionierten Krediten profitierten, verringerten die Verschuldung besonders stark. Die Schätzungen zeigen, dass der Rückgang der Bankkredite (im Verhältnis zur Bilanzsumme) in jenen Branchen um 7 Prozentpunkte stärker ausfiel als in nur schwach bankabhängigen. Auch die Kreditvergabe an unprofitable Unternehmen ging deutlich zurück: Unternehmen mit besonders niedriger Rentabilität reduzierten ihren Verschuldungsgrad von 55 auf $40 \%$, und damit mehr als doppelt so stark als der Durchschnitt (48 auf $42 \%$ ). Teilweise wurde der Rückgang des Fremdkapitals durch Handelskredite oder die Aufnahme von Eigenkapital ausgeglichen. Da sich im selben Zeitraum allerdings die Zinsen erhöht haben, ist nicht genau zu beziffern, welcher Anteil dieser Veränderungen auf die Reform und welcher auf die Zinsen zurückzuführen ist.

Die Reform des Bankensektors hatte realwirtschaftliche Konsequenzen. Industriezweige, welche besonders stark von Bankkrediten abhängig waren, wurden kosteneffektiver und wuchsen weniger stark. Diese Entwicklung deutet auf Überinvestitionen vor der Reform hin. Die Schrumpfung führte also zu einer effizienteren 
Nutzung von Ressourcen und zu höheren Renditen. Der Anpassungsprozess hatte allerdings auch schmerzhafte Folgen. So sanken die Löhne relativ zu den Branchen, die sich weniger über Bankkrediten finanzierten.

Die Deregulierung führte in besonders bankabhängigen Sektoren zu einem Rückgang der Bilanzsumme relativ zu anderen Sektoren um $6 \%$ und der Löhne um $4 \%$.

Die Ergebnisse beziehen sich jeweils auf Branchendurchschnitte. Es stellt sich die Frage, ob bestehende Unternehmen ihre Effizienz verbessert haben, oder ob die Effizienzsteigerungen mehr auf das Entstehen von jungen, effizienten Unternehmen und dem Ausscheiden von besonders unprofitablen Firmen zurückgehen. Nach der Deregulierung des Bankensektors nahm die Zahl von Unternehmensgründungen, aber auch von Schließungen zu. Die damit verbundene Fluktuation von Arbeitskräften und Kapital stieg überdurchschnittlich stark in Branchen, welche vor der Reform besonders abhängig vom Bankensektor waren und danach einen aufgestauten Bedarf an Reallokation bewältigen mussten. In diesen Branchen ging auch die Marktkonzentration zurück. Die Marktanteile einzelner großer Unternehmen schrumpften und verteilten sich auf eine größere Anzahl von Firmen. Eine geringere Marktkonzentration bedeutet stärkeren Wettbewerb und wirkt sich tendenziell für die Konsumenten positiv aus. Die Autoren schlossen aus diesen Ergebnissen, dass die staatlichen Interventionen Unternehmensgründungen erschwerten und die etablierten Unternehmen bevorteilten.

Nach der Reform stiegen die Markteintritte gemessen an den neu geschaffenen Vermögenswerten in besonders bankabhängigen Sektoren um $26 \%$ stärker an.

Ein leistungsfähiger Bankensektor spielt somit eine wichtige Rolle, Innovation und Wachstum durch „schöpferische Zerstörung“, wie es der österreichische Ökonom Josef Schumpeter nannte, voranzutreiben. Die Aufgabe alter Technologien und die Umlenkung von Arbeit und Kapital auf neue innovative Branchen ist dabei ein Motor des Wirtschaftswachstums und Fortschritts. Die Ergebnisse der Forscher deuten darauf hin, dass die starken staatlichen Eingriffe im Bankensektor letzten Endes die alteingesessenen Unternehmen vor der effizienzfördernden Konkurrenz neuer Markteintritte geschützt und damit Frankreichs Wachstum gebremst haben. 
Open Access Dieses Kapitel wird unter der Creative Commons Namensnennung 4.0 International Lizenz (http://creativecommons.org/licenses/by/4.0/deed.de) veröffentlicht, welche die Nutzung, Vervielfältigung, Bearbeitung, Verbreitung und Wiedergabe in jeglichem Medium und Format erlaubt, sofern Sie den/die ursprünglichen Autor(en) und die Quelle ordnungsgemäß nennen, einen Link zur Creative Commons Lizenz beifügen und angeben, ob Änderungen vorgenommen wurden.

Die in diesem Kapitel enthaltenen Bilder und sonstiges Drittmaterial unterliegen ebenfalls der genannten Creative Commons Lizenz, sofern sich aus der Abbildungslegende nichts anderes ergibt. Sofern das betreffende Material nicht unter der genannten Creative Commons Lizenz steht und die betreffende Handlung nicht nach gesetzlichen Vorschriften erlaubt ist, ist für die oben aufgeführten Weiterverwendungen des Materials die Einwilligung des jeweiligen Rechteinhabers einzuholen.

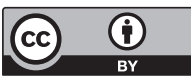

